\title{
Spatial heterogeneity of oxygenation and haemodynamics in breast cancer resolved in vivo by conical multispectral optoacoustic mesoscopy
}

\author{
Jiao Li $i^{1,2,3}$, Andrei Chekkoury ${ }^{2,3}$, Jaya Prakash ${ }^{2,3,4}$, Sarah Glas| ${ }^{2,3}$, Paul Vetschera ${ }^{2,3}$, Benno Koberstein-Schwarz ${ }^{2,3}$, \\ Ivan Olefir ${ }^{2,3}$, Vipul Gujrati ${ }^{2,3}$, Murad Omar $\mathbb{E}^{2,3}$ and Vasilis Ntziachristos $\mathbb{E}^{2,3}$
}

\begin{abstract}
The characteristics of tumour development and metastasis relate not only to genomic heterogeneity but also to spatial heterogeneity, associated with variations in the intratumoural arrangement of cell populations, vascular morphology and oxygen and nutrient supply. While optical (photonic) microscopy is commonly employed to visualize the tumour microenvironment, it assesses only a few hundred cubic microns of tissue. Therefore, it is not suitable for investigating biological processes at the level of the entire tumour, which can be at least four orders of magnitude larger. In this study, we aimed to extend optical visualization and resolve spatial heterogeneity throughout the entire tumour volume. We developed an optoacoustic (photoacoustic) mesoscope adapted to solid tumour imaging and, in a pilot study, offer the first insights into cancer optical contrast heterogeneity in vivo at an unprecedented resolution of $<50 \mu \mathrm{m}$ throughout the entire tumour mass. Using spectral methods, we resolve unknown patterns of oxygenation, vasculature and perfusion in three types of breast cancer and showcase different levels of structural and functional organization. To our knowledge, these results are the most detailed insights of optical signatures reported throughout entire tumours in vivo, and they position optoacoustic mesoscopy as a unique investigational tool linking microscopic and macroscopic observations.
\end{abstract}

\section{Introduction}

Tumours contain genetically and phenotypically distinct cell subpopulations exposed to heterogeneous vascularization and signalling microenvironments ${ }^{1}$. Understanding the tumour microenvironment and its heterogeneity are key to elucidating cancer progression, metastasis and response to treatment. In addition to genetic heterogeneity, spatial heterogeneity may play a vital role in tumour growth, metastasis or treatment, especially if different parts of a tumour respond in different ways ${ }^{2}$.

Correspondence: Vasilis Ntziachristos (v.ntziachristos@tum.de)

${ }^{1}$ School of Precision Instruments and Optoelectronics Engineering, Tianjin University, No.92, Weijin Road, Nankai District, 300072 Tianjin, China

${ }^{2}$ Institute of Biological and Medical Imaging, Helmholtz Zentrum München, Ingolstädter Landstr. 1, D-85764 Neuherberg, Germany

Full list of author information is available at the end of the article
Intravital (in vivo) microscopy is commonly used in cancer research and has revealed critical information about cell motility that leads to metastasis, about the function of immune cells within tumours and their role in inhibiting or promoting tumour growth, and about the different responses of cell populations within tumours to the same drug ${ }^{3-5}$ or to radiation therapy ${ }^{6}$. Nevertheless, optical microscopy is limited by photon scattering ${ }^{7}$ and can assess small volumes of only a few hundred cubic microns ${ }^{1,8}$, so it is not suited for comprehensively studying the morphology, functionality and the overall heterogeneity of an entire tumour volume9. Surgical removal of tissue and the implantation of "optical windows"10 allow microscopic observations of deeper tumour areas but similarly limit the comprehensive visualization of entire tumours. The need to image larger volumes has led to the development of several alternatives. Three-

\section{(c) The Author(s) 2020}

(c) (i) Open Access This article is licensed under a Creative Commons Attribution 4.0 International License, which permits use, sharing, adaptation, distribution and reproduction c. in any medium or format, as long as you give appropriate credit to the original author(s) and the source, provide a link to the Creative Commons license, and indicate if changes were made. The images or other third party material in this article are included in the article's Creative Commons license, unless indicated otherwise in a credit line to the material. If material is not included in the article's Creative Commons license and your intended use is not permitted by statutory regulation or exceeds the permitted use, you will need to obtain permission directly from the copyright holder. To view a copy of this license, visit http://creativecommons.org/licenses/by/4.0/. 
dimensional light-sheet microscopy ${ }^{11,12}$ offers the possibility of volumetric imaging. However, it requires chemical processing of specimens to make them transparent and is therefore not suited for in vivo applications or imaging of tissue physiology.

As an alternative to optical methods, optoacoustic imaging has demonstrated high-resolution imaging of optical contrast through several millimetres to centimetres of tissue ${ }^{13-16}$. There are two major optoacoustic imaging techniques. Optical-resolution optoacoustic (photoacoustic) microscopy uses focused light to excite ultrasound responses, but its depth and resolution are similar to those of optical (photonic) microscopy since both modalities form images limited by optical diffraction. Conversely, acoustic-resolution optoacoustic imaging can be performed using unfocused illumination, in which case the depth and resolution depend strongly on the characteristics of the ultrasound detection arrangement employed and the ultrasound frequency band collected. The combination of deep-seated high-resolution imaging with multi-wavelength illumination gives rise to multispectral optoacoustic tomography (MSOT), which can resolve the spectral signatures of intrinsic chromophores and extrinsically administered absorbers. MSOT has been consequently employed to visualize and quantitate the oxygenation status of tumours ${ }^{17-19}$ and resolve angiogenesis and a number of external agents targeting physiological and molecular tumour parameters in animals and humans ${ }^{19-25}$. Nevertheless, current optoacoustic imaging systems do not allow high-resolution imaging of tumour heterogeneity. Tomographic systems using detector arrays have allowed three-dimensional imaging that yielded cross-sectional images throughout entire tumours ${ }^{18}$, but the resolutions achieved have been on the order of $100-500 \mu \mathrm{m}$. These resolutions prevent the detailed observation of tumour spatial heterogeneity. Systems that raster scan an ultrasound detector operating at higher frequencies than detector arrays allow higher resolution imaging but offer only limited-view images of superficial vasculature $20,21,23,24,26,27$, typically rendered as two-dimensional maximum intensity projections (MIPs).

Herein, we introduce multispectral optoacoustic mesoscopy (MSOM) with a novel system that combines threedimensional performance with high-resolution imaging. We identified $\sim 1-30 \mathrm{MHz}$ as the optimal ultrasound detector bandwidth offering the optimal trade-off between resolution and cross-sectional imaging of entire tumours. We implemented this frequency band in a conical geometry enabling optical views of tumour heterogeneity with unprecedented detail. By applying MSOM at multiple wavelengths, we studied, for the first time, patterns of the spatial heterogeneity of tumour oxygenation and validated these patterns through co-registration with patterns of vascularization and permeability throughout the entire tumour mass. By applying the technique to three breast cancer models in mice, we studied intra- and inter-tumour heterogeneity and showed how in vivo MSOM imaging can be applied to quantitatively characterize the spatial heterogeneity in breast cancer based on functional tumour parameters in vivo. We discuss the unique performance achieved by our conical MSOM implementation and the implications for providing new possibilities in cancer research.

\section{Results}

The MSOM system employed (Fig. 1a) is a secondgeneration mesoscopy setup with key innovations that allow in vivo visualization throughout entire solid tumours. The system uses four-sided illumination, and the generated acoustic signal is detected using a 96element linear array detector with a central frequency of $15 \mathrm{MHz}$ and bandwidth of $\sim 1-30 \mathrm{MHz}$. This bandwidth was found to be optimal for imaging volumes measuring $5-10 \mathrm{~mm}$ in diameter, according to results from simulations and experimentation with other frequencies in the past $^{28-30}$. A further novelty of the system is the combination of four-sided illumination and a conical geometry, in which the detector is positioned at a 45-degree angle to the rotation plane, thus allowing the accommodation of tumours of different sizes (Fig. 1a). MSOM imaging of tumours was performed at five wavelengths to capture the distributions of different intrinsic chromophores, such as deoxygenated haemoglobin $(\mathrm{Hb})$ and oxygenated haemoglobin $\left(\mathrm{HbO}_{2}\right)$, and of extrinsic probes such as gold nanoparticles (Fig. 1c).

Images were obtained by collecting data in translationrotation conical scanning mode. Compared to rotationonly or translation-only scanning, translation-rotation scanning has previously demonstrated superior mesoscopic imaging performance ${ }^{31}$. Reconstruction was based on a backprojection algorithm ${ }^{31}$ adapted to the conical geometry. Previously reported methods for image reconstruction at different frequency bands and frequency equalization were borrowed from raster scan optoacoustics $^{32}$ and applied for the first time in MSOM. We separated a low-frequency $(1-7.5 \mathrm{MHz})$ and high-frequency (4-28 MHz) band (Fig. 1d-i). Band-specific reconstruction and frequency equalization have been shown to provide better signal-to-noise ratio and rendering than single-band reconstruction ${ }^{32}$, especially in regard to the high-spatial-frequency components (fine details) (SI Appendix, Fig. S1).

The resolution of the MSOM system was characterized using $20 \mu \mathrm{m}$ polyethylene microspheres dispersed in an agar cylinder. The system resolved spheres with a full width at half-maximum diameter of $62 \mu \mathrm{m}$ for fullfrequency band reconstruction (Fig. 1b), indicating an in-plane system resolution of $\sim 50 \mu \mathrm{m}$ after deconvolution 


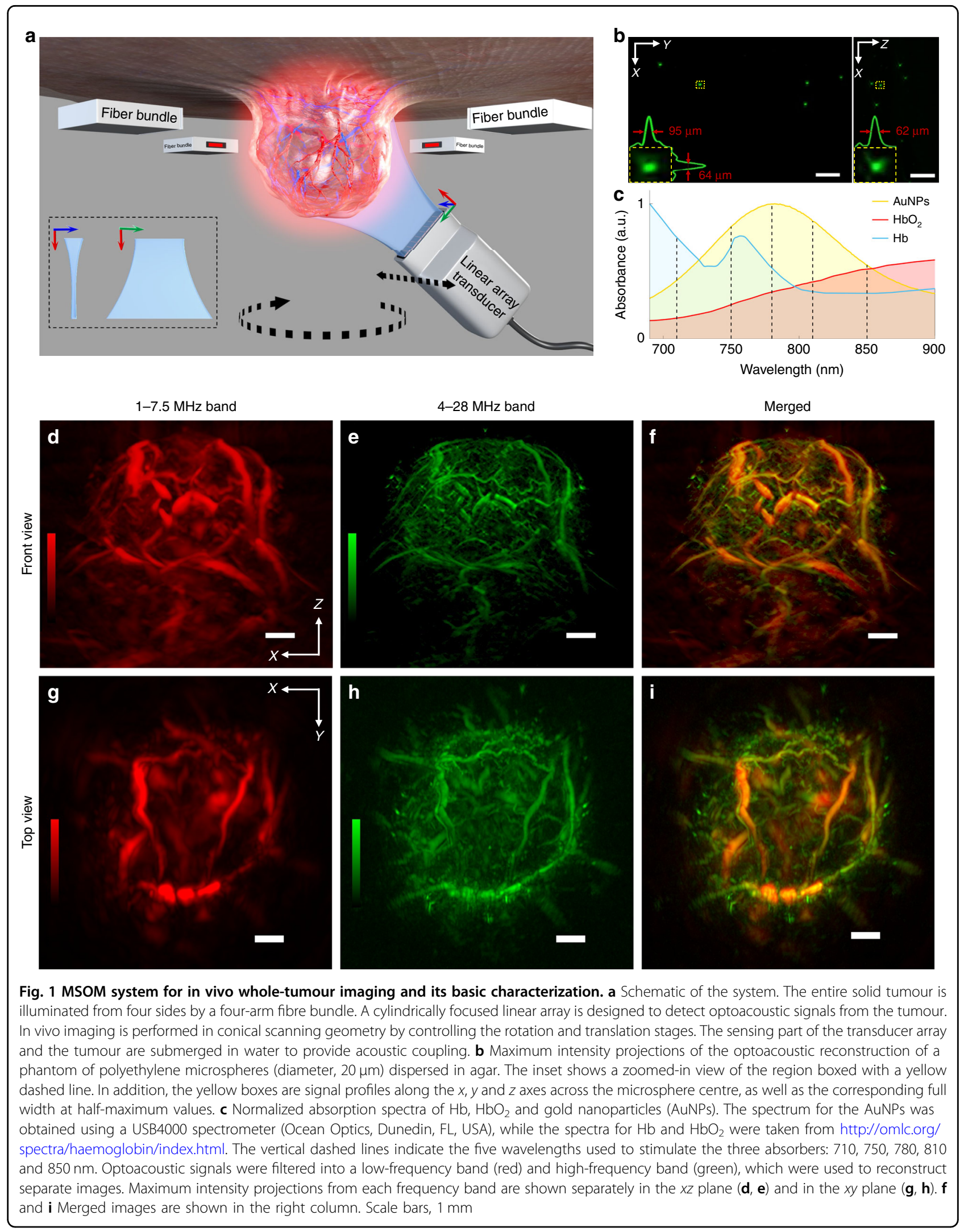


of the finite sphere size and total impulse response through at least $\sim 1 \mathrm{~cm}$ of tissue (SI Appendix, Fig. S2a, d). The band-specific resolution was $\sim 38 \mu \mathrm{m}$ for the highfrequency band and $\sim 92 \mu \mathrm{m}$ for the low-frequency band, as also shown in SI Appendix, Fig. S2.

MSOM was used to interrogate differences in the spatial heterogeneity of total haemoglobin concentration $(\mathrm{HbT}=$ $\left.\mathrm{Hb}+\mathrm{HbO}_{2}\right)$, oxygen saturation $\left(\mathrm{sO}_{2}=\mathrm{HbO}_{2} / \mathrm{HbT}\right)$ and vascular permeability. $\mathrm{HbT}$ and $\mathrm{sO}_{2}$ were computed by resolving the distribution of $\mathrm{Hb}$ and $\mathrm{HbO}_{2}$ in entire tumours in vivo at resolutions never before possible. The spatial heterogeneity of these parameters was quantitated throughout the volumes of three types of breast tumours: two human breast cancer xenografts (KPL4, MDA-MB231) and one mouse mammary tumour allograft (4T1). Vascular permeability in 4T1 tumours in mice following injection of gold nanoparticles was also studied.

Figure 2 shows the distribution of $\mathrm{Hb}, \mathrm{HbO}_{2}, \mathrm{HbT}$ and $\mathrm{sO}_{2}$ throughout a $4 \mathrm{~T} 1$ tumour with a diameter of $8 \mathrm{~mm}$ growing within the mouse mammary fat pad. The images demarcate the tumour boundaries and details of the vascular networks and heterogeneity patterns attributed to optical contrast throughout the tumour. Two tumour representations are shown. One depicts MIPs of the entire tumour in the axial dimension, as shown in the four large panels in Fig. 2. This representation provides a holistic view of the tumour volume and primarily reveals large peripheral feeder blood vessels surrounding the tumour surface, which appear to be connected with microvessels within the tumour. Two-band frequency equalization enables better separation of large vessels from smaller ones (see Methods and Fig. 1d-i). Extensive highly oxygenated areas are visible on the tumour periphery, while areas of lower oxygenation are visible in the tumour core. The second tumour representation, as shown in the smaller panels marked I-IV in Fig. 2, provides crosssectional (coronal) views throughout the tumour mass. Four coronal slices of $400 \mu \mathrm{m}$ thickness each are shown. This type of cross-sectional image showcases the unique abilities of MSOM, allowing the first observations of spatial patterns of $\mathrm{HbT}$ and $\mathrm{sO}_{2}$ within an entire tumour at resolutions previously inaccessible to in vivo optical imaging. Spectral error analysis highlights the high signalto-noise ratio obtained in the images shown (SI Appendix, Fig. S3).

Tumours that were imaged in vivo were also processed ex vivo. The tumours were harvested from euthanized mice and preserved in tissue-freezing medium at $-80{ }^{\circ} \mathrm{C}$. Interleaved tumour tissue slices were stained with haematoxylin-eosin (H\&E), anti-CD31 antibody for vascular endothelial cell staining ${ }^{33}$ and anti-HIF- $1 \alpha$ antibody for identifying hypoxic areas in the tumour core ${ }^{34}$. The MSOM slices shown are of different thicknesses than the histology images. Moreover, the registration of MSOM images obtained in vivo and histological images obtained ex vivo with a different imaging system is only approximate. Nevertheless, comparison of MSOM slices and histological slices from approximately the same area/ volume of the tumour (Fig. 3) revealed correspondence between the recorded signals. HbT-MSOM images corresponding to endothelial marker CD31 staining reveal the vasculature density, average radius (vessel size) and blood vessel distribution ${ }^{25}$ (Fig. 3a, d). Inspection revealed that the distribution of $\mathrm{Hb}$ correlated with immunohistochemical staining for hypoxia-associated HIF-1 $\alpha$ (Fig. $3 \mathrm{~b}, \mathrm{e})$. Profiles are obtained through images along the white lines (Fig. 3a, d) and then processed using the moving window averaging method. The profiles show similar variations in spatial patterns across the tumour (Fig. 3g, h) (see Methods). Overall, fluorescence micrographs show more abundant HIF- $1 \alpha$ in the tumour core than in the middle region, consistent with the greater hypoxia in the core (SI Appendix, Fig. S4). Finally, we also compared MSOM images of the oxygenated haemoglobin distribution (Fig. 3c) to cryosections of the tumour (Fig. 3f) and H\&E staining images of select regions (Fig. 3i, j). Figure $3 \mathrm{f}$ shows that the redder regions in the cryosection image corresponded to areas with increased oxygenated haemoglobin levels. The images in Fig. 3i, $j$ are of H\&Estained microscopy slices taken from the areas indicated in Fig. 3c, $\mathrm{f}$ with the green and blue arrows, respectively. The H\&E images show the different densities of tumour cells in regions of varying $\mathrm{HbO}_{2}$ concentrations. Figure $3 \mathrm{k}-\mathrm{m}$ reveals further correspondence between the MSOM and histology results through a histogram and Hamming distance analysis of the images (see Methods). The Hamming distance was calculated by a perceptual image hash function (pHash) in MATLAB ${ }^{35}$. While the analysis in Fig. 3 is of slices of the 4T1 tumours, results obtained from KPL4 and MDA-MB-231 tumours were also validated histologically (SI Appendix, Figs. S5, 6).

Figure $4 \mathrm{a}-\mathrm{c}$ extends the observations of tumour heterogeneity by comparing cross-sectional (coronal) images obtained throughout the tumour volume for all three tumour types (4T1, KPL4 and MDA-MB-231). These images allow a differential observation of $\mathrm{HbT}$ and $\mathrm{sO}_{2}$ patterns across different tumour types, pointing to the unique potential of MSOM to capture intra- and intertumour heterogeneity. Visual observation preliminarily revealed qualitative differences across tumours, with the MDA tumour exhibiting less optical contrast in its core and less superficial vasculature than the other tumours.

To quantitatively process and understand the heterogeneity patterns observed, we studied the Tamura metrics of coarseness, contrast and directionality ${ }^{36}$ as well as general metrics such as average intensity per region of interest, variance, skewness, kurtosis and energy based on histogram or statistical distributions ${ }^{37}$ (SI Appendix, 


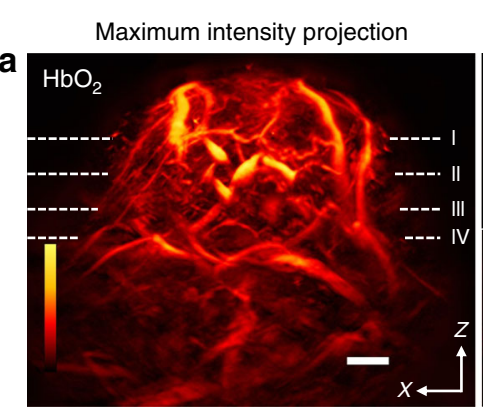

Cross-sectional slices
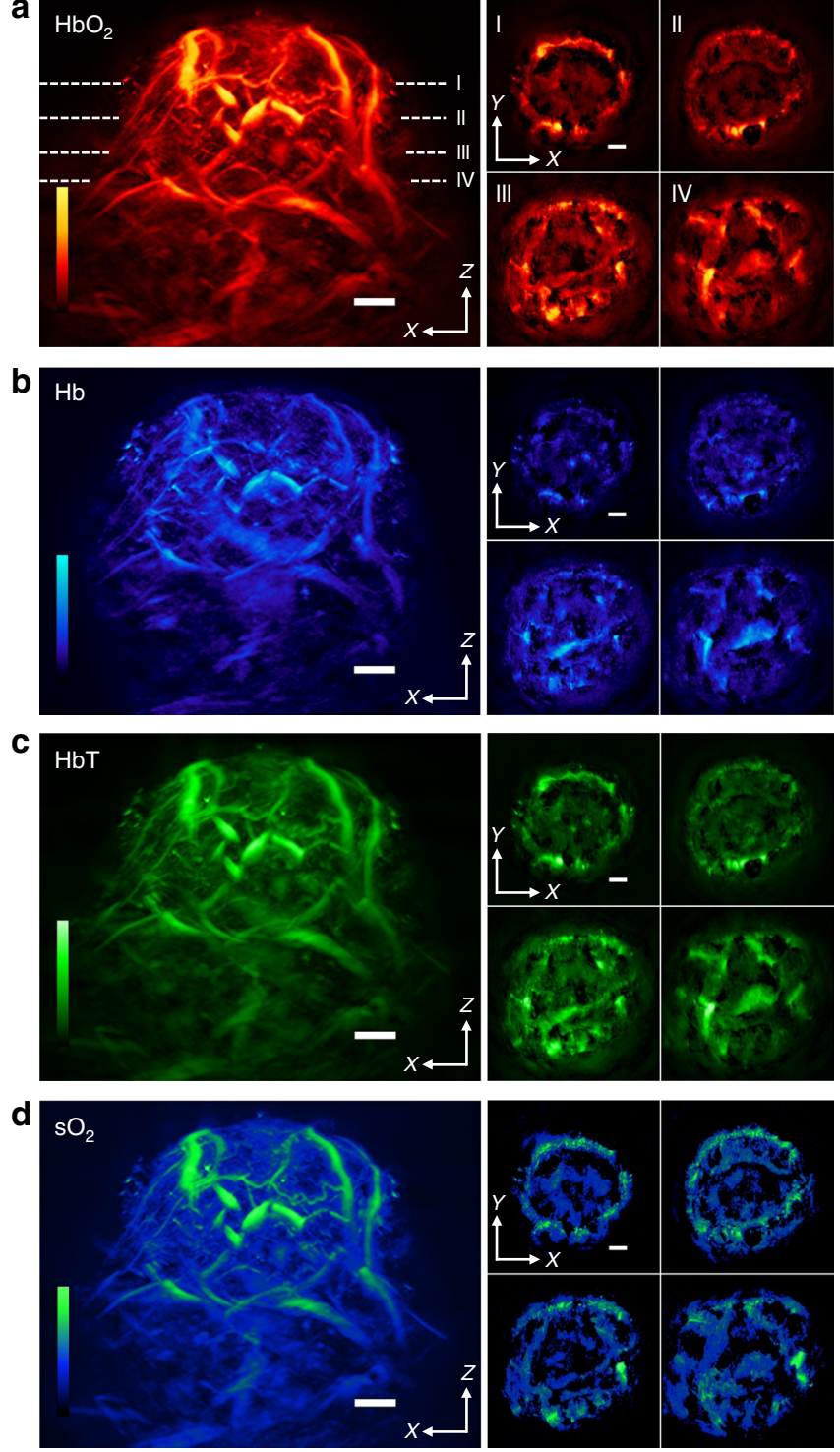

Fig. 2 Whole-tumour in vivo MSOM imaging based on endogenous $\mathbf{H b}$ and $\mathbf{H b O}_{2}$ contrast. Representative results are shown for one $4 \mathrm{~T} 1$ tumour. The larger panels show maximum intensity projections in the $x z$ plane for $(\mathbf{a}) \mathrm{HbO}_{2},(\mathbf{b}) \mathrm{Hb}$, (c) total haemoglobin concentration ( $\left.\mathrm{HbT}\right)$, and (d) oxygen saturation $\left(\mathrm{s}_{2}\right)$. Next to each larger panel are maximum intensity projections in the xy plane corresponding to the four tumour depths (I-IV). These four tumour sections compose the entire tumour depth. Maximum intensity projections were calculated over tumour sections with a thickness of $400 \mu \mathrm{m}$. Scale bars, $1 \mathrm{~mm}$

Table S1). These traditional metrics have been previously applied to texture analysis of tumour heterogeneity using imaging modalities such as CT, MRI and $\mathrm{PET}^{38-44}$. For example, higher variance indicates greater dispersion around the average, corresponding to the presence of more highlighted objects with greater intensity differences from the background. Kurtosis indicates how irregular or flat the intensity histogram is relative to a Gaussian distribution. Kurtosis increases when pixel intensity is much brighter or darker than the background, but it decreases as the number of brighter or darker pixels increases ${ }^{43}$. This may make it an indicator of the vasculature complexity or necrosis degree. Skewness, which reflects the asymmetry of the histogram, refers to the average brightness of highlighted objects surrounded by background or less intense regions. Positive skew values indicate the presence of bright objects; negative skew values, the presence of dark objects. Contrast, which reflects variations between neighbouring pixels, relates to the dynamic range of intensity level in the image and may 


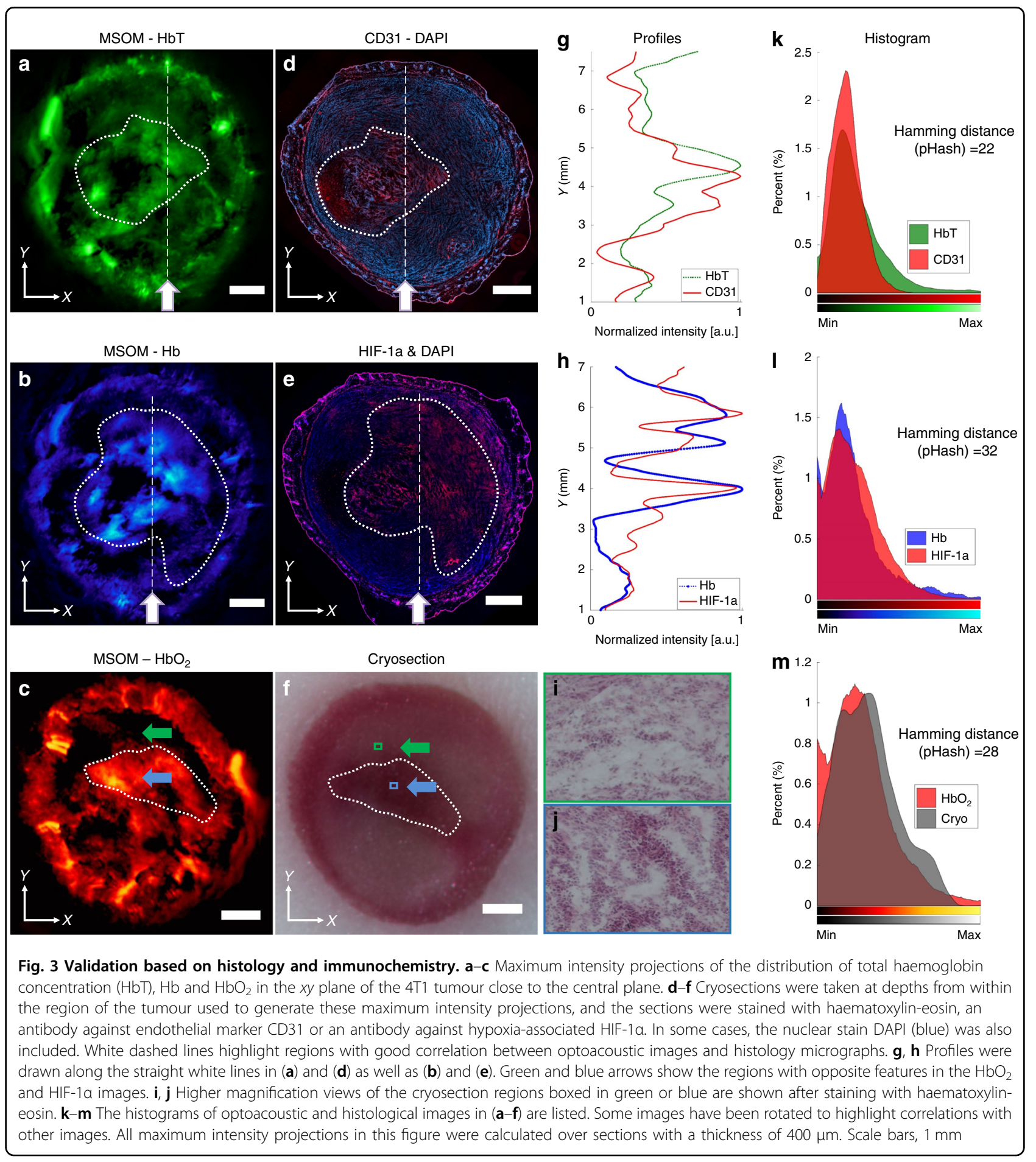

also indicate the extent of tumour heterogeneity. Variance, as an indicator of the number of dark or bright tumour regions corresponding to hypoxic areas or areas of high angiogenesis, may correlate directly with tumour heterogeneity. As shown in SI Appendix, Table S1, contrast is proportional to the square of variance, while kurtosis and skewness are inversely proportional to the third and fourth power of variance, respectively. In Fig. $4 \mathrm{~d}-\mathrm{k}$, the metrics of both $\mathrm{HbT}$ and $\mathrm{sO}_{2}$ reflect the same correlations with their mathematical relations.

Figure $4 \mathrm{~d}-\mathrm{g}$ shows various metrics of $\mathrm{sO}_{2}$ and $\mathrm{HbT}$ heterogeneity on the whole-tumour level, while Fig. $4 \mathrm{~h}-\mathrm{k}$ shows metrics of heterogeneity separately for the tumour boundary (rim) and tumour core (centre). The results 


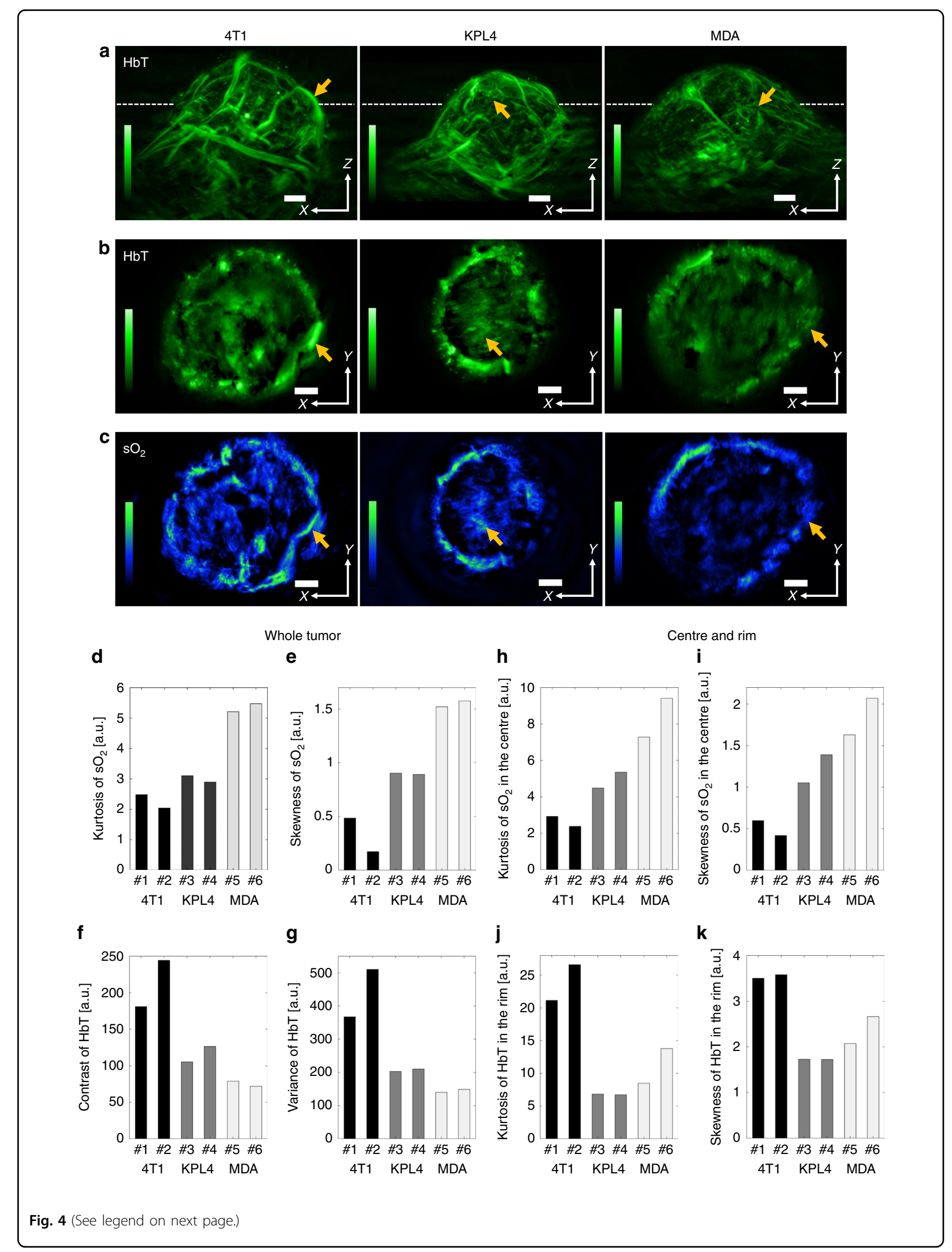


(see figure on previous page)

Fig. 4 Comparison of in vivo MSOM images of three breast cancer tumour types and MSOM-based in vivo quantitation of $\mathrm{SO}_{2}$ and $\mathrm{HbT}^{\mathrm{B}}$ heterogeneity throughout entire tumours. a The upper row shows representative maximum intensity projections of total haemoglobin concentration $(\mathrm{HbT})$ in the $x z$ plane of entire tumours in vivo. b, c Maximum intensity projections of the distribution of (b) $\mathrm{HbT}^{\mathrm{b}}$ and $(\mathbf{c}) \mathrm{sO}_{2}$ in the $x y$ plane of the tumours, as defined by the dashed lines in panel (a). $\mathbf{d}-\mathbf{k}$ Various heterogeneity metrics were measured in two biological replicates of the three types of breast cancer tumours. Details of how metrics were calculated can be found in the Methods section, together with an explanation of how tumours were segmented into centre and rim regions. MDA refers to MDA-MB-231. Some images have been rotated to highlight correlations with other images. All maximum intensity projections in this figure were calculated over sections with a thickness of $400 \mu \mathrm{m}$. Scale bars, $1 \mathrm{~mm}$

illustrate how MSOM can provide global and local quantitative functional information in a single noninvasive experiment. One intriguing result, for example, is that the KPL4 and MDA-MB-231 tumours showed higher $\mathrm{sO}_{2}$ kurtosis and skewness than 4T1 tumours (cf. panels $\mathrm{d}-\mathrm{e}$ ) and that this difference was even more apparent in the tumour centre than on the tumour rim (cf. panels h-i). Although the $\mathrm{sO}_{2}$ contrast and variance in 4T1 tumours were close to those in the two other tumour types, as shown in SI Appendix, Fig. S7c, d, the smaller differences in heterogeneity between the centre and rim in 4T1 tumours than in the tumours of the other types, as shown in SI Appendix, Fig. S7g, h, raises the possibility that 4T1 tumours are less heterogeneous in $\mathrm{sO}_{2}$ and less hypoxic overall than KPL4 or MDA-MB-231 tumours. SI Appendix, Fig. S8 shows the heterogeneity in $\mathrm{HbT}$ rather than $\mathrm{sO}_{2}$ for the three tumour types. As in the case of $\mathrm{sO}_{2}$, $\mathrm{HbT}$ heterogeneity varied substantially across tumour types. 4T1 tumours showed greater heterogeneity than the other two tumour types, and this heterogeneity may be present more in the rim than in the centre. These results provide the first evidence that MSOM can be employed to assess morphological and physiological differences within the tumour mass. Other heterogeneity metrics analysed at the whole-tumour level are listed in SI Appendix, Fig. S9. The MSOM-based in vivo quantitation of $\mathrm{sO}_{2}$ and $\mathrm{HbT}$ heterogeneity in individual $x y$ plane sections of all tumour types and depths (SI appendix, Fig. S10) was comparable to that at the whole-tumour level (Fig. 4d-k).

To explore the ability of MSOM to capture vascular permeability, we intravenously injected gold nanoparticles into a mouse bearing a 4T1 tumour. Such nanoparticles have been used for optoacoustic contrast enhancement and theranostics ${ }^{45,46}$. The mouse was imaged with MSOM throughout the entire tumour mass at 1 and $24 \mathrm{~h}$ after injection. Figure 5 shows that by $1 \mathrm{~h}$, the optoacoustic signal due to the nanoparticles appeared primarily along the tumour boundary, and minimal signal was present within the tumour core; by $24 \mathrm{~h}$, a substantial nanoparticle signal was present throughout the tumour core. Figure 5 shows nanoparticle distribution maps obtained for volumetric sections taken at two tumour depths, where the changes at different time points of perfusion progress can be obviously observed, especially in the comparison of various heterogeneity metrics between the centre and boundary of the tumour. Compared to the heterogeneity metrics at $1 \mathrm{~h}$ after injection, the metrics of the tumour centre approach or even exceed those of the tumour boundary at $24 \mathrm{~h}$ after injection. These results illustrate the ability of MSOM to track the penetration of nanoparticles as they move from the tumour periphery to the core. These results were validated by performing darkfield microscopy of tissue cryosections, in which a substantial gold nanoparticle signal was observed in the tumour core (SI Appendix, Fig. S11). SI Appendix, Fig. S12 shows the nanoparticle distribution in the three breast cancer tumour types at $24 \mathrm{~h}$ after injection. The 4T1 tumour shows higher nanoparticle intensity in the centre than do the other two tumour types. The kurtosis, skewness, contrast and variance metrics varied across the three tumour types, especially when we took the ratio of the tumour centre to the tumour boundary. The four metrics show similar values at the centre and boundary in the case of the MDA tumour but different values at the centre and boundary in the case of the 4T1 and KPL4 tumours. In fact, the two tumour regions differ in opposite directions between 4T1 and KPL4 tumours.

\section{Discussion}

For the first time, we characterized the spatial heterogeneity of tumour hypoxia $\left(\mathrm{sO}_{2}\right)$ and the corresponding vascular patterns throughout entire tumours in vivo at resolutions never before demonstrated by another method. By doing so, we also demonstrated that by using optoacoustic imaging, we can observe different patterns between tumours, allowing study of their individualized morphology and functions.

Focal hypoxia of solid tumours has been considered a prognostic biomarker and driver of tumour progression because of its connection with poor patient outcomes and therapy resistance ${ }^{1}$. Therefore, quantitating hypoxia in this way may provide valuable information for screening drug candidates and assessing patient response to therapy $^{19}$. Segmented quantitation of HbT further allowed us to examine vascularization in different parts of the 


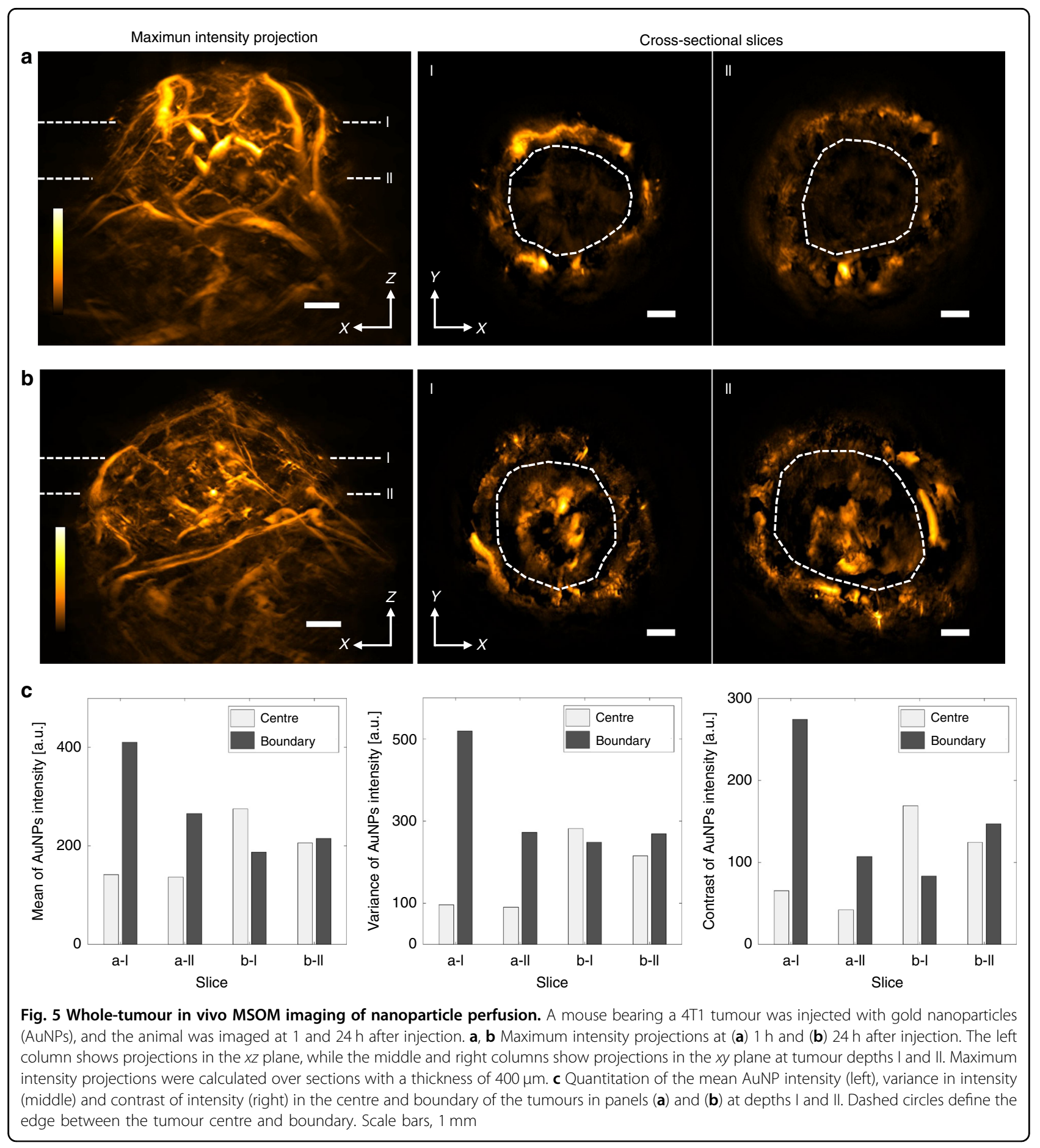

tumour, demonstrating divergent heterogeneous patterns. Such analyses of $\mathrm{sO}_{2}$ and $\mathrm{HbT}$, which can assess the hypoxic microenvironment and vessel abnormalities of solid tumours, may provide a platform for the in vivo assessment of emerging anticancer therapies aimed at alleviating hypoxia and inhibiting tumour angiogenesis $^{1,47}$. Poor tumour oxygenation has been linked to treatment resistance and worse outcomes ${ }^{19}$.
Overall, this study revealed that significant changes in optical contrast exist in different parts of the tumour. In particular, the tumour boundary showed angiogenesis and oxygenation patterns that differ significantly from those in the tumour core. However, this heterogeneity is not entirely predictable: for example, the tumour core also contains foci of vascularization and oxygenation intermingled with areas of severe hypoxia. For this reason, 
intravital microscopy, although it reveals sub-micrometre details of tumour biology, can provide only a local, fragmented understanding of tumour structure and function. MSOM, by covering a volume three orders of magnitude larger than intravital microscopy ( 50 vs. $0.5 \mu \mathrm{m})$, provides a holistic view of tumours. In this way, the two techniques are highly complementary: MSOM can provide an overall view, within which specific regions can be analysed at higher resolution using intravital microscopy.

The results of this pilot study on technology feasibility clearly demonstrated differences in the heterogeneity present in different tumours. The study shows that the novel $15 \mathrm{MHz}$ MSOM system can indeed interrogate the variability between tumour types and explore the relation between tumour type and heterogeneity in morphological and functional parameters such as vascularization and $\mathrm{sO}_{2}$ on a tumour-to-tumour basis. The relatively high quality of the images provided by the MSOM system described here likely reflects critical improvements over our previously published design ${ }^{28}$. The linear transducer array with a central frequency of $15 \mathrm{MHz}$ can capture acoustic signals at a high signal-to-noise ratio from lightabsorbing anatomical structures in the tumour smaller than $100 \mu \mathrm{m}$ (blood vessels) up to structures spanning several millimetres (tumour core). The tumour in our system was illuminated with broad-field illumination from four sides, helping provide homogeneous stimulation of light absorbers in the tumour, leading in turn to stronger and more uniform acoustic signals despite depthdependent attenuation of light fluence. The customdesigned sample holder features a tumour hole with plastic snap rings of various inner diameters that can be optimized to the tumour size. In addition, a linear stage allows the sample holder to be raised or lowered to position the sample optimally within the field of view of the detector. Frequency equalization, in which the relative contributions of acoustic signals of different frequencies are balanced, improves image fidelity by recovering chromophores of various sizes in the tumour, such as smaller and larger vessels. This advanced performance was essential to provide a detailed analysis of and insights into tumour patterns.

Our pilot study suggests that 4T1 may be less heterogeneous in $\mathrm{SO}_{2}$ and less hypoxic than KPL4 or MDA-MB231 tumours; nevertheless, as a future step, it would be interesting to investigate these findings in a larger tumour sample in the context of explaining the particularly aggressive growth and multi-site metastasis of $4 \mathrm{~T} 1$ tumours $^{48}$. Tumour physiological heterogeneity and, in particular, oxygenation parameters can only be resolved at resolution orders of magnitude lower using nuclear imaging techniques, which require radioactivity and thereby limit possibilities for longitudinal studies. The possibility of using high-resolution MSOM to analyse tumour heterogeneity may create new opportunities for studying tumour heterogeneity in a more detailed manner. Indeed, MSOM fulfils one set of proposed requirements ${ }^{49}$ for analysing three-dimensional variations in tumour properties: high spatial resolution $(10-100 \mu \mathrm{m})$ and a unique contrast and feature set not available to other imaging modalities. The study suggests the potential of textural measures of spatial heterogeneity such as skewness and kurtosis for image-based analysis of breast cancer. Skewness describes tumour characteristics, and in our samples, more extensive angiogenesis (4T1) was associated with greater positive skew values than less extensive angiogenesis (MDA and KPL4). The kurtosis values in our samples were inversely proportional to noise. The contrast and variance in our samples correlated negatively with hypoxia but positively with angiogenesis, which can be seen in the $\mathrm{sO}_{2}$ difference between the tumour centre and rim and in the $\mathrm{HbT}$ values at the rim. Measures of heterogeneity such as kurtosis are employed in other imaging modalities, such as X-ray $\mathrm{CT}^{44,50}, \mathrm{PET}^{39,50}$ or $\mathrm{MRI}^{41}$, for assessing tumour malignancy or staging ${ }^{51-56}$, therapeutic response and efficacy ${ }^{41,57}$ and survival outcomes $^{58-61}$. In this study, we employed these metrics for the first time in analysing optical contrast in high resolution, showcasing previously undisclosed optical and $\mathrm{sO}_{2}$ variability between the tumours studied. Compared to earlier work on tissue heterogeneity, such as spectrum analysis approaches ${ }^{62-64}$ and MSOT $^{18}$, our work focused on providing a mesoscopic tool operating at a centre frequency of $15 \mathrm{MHz}$ to offer in vivo three-dimensional functional images with resolutions of $38-92 \mu \mathrm{m}$ throughout whole solid tumours. Therefore, MSOM allows us to observe fine details of cancer haemodynamic contrast that go beyond the intensity and frequency signals of optoacoustic images obtained at macroscopic resolutions.

Uniquely among high-resolution imaging techniques, MSOM can visualize tissue features based on endogenous contrast from $\mathrm{Hb}, \mathrm{HbO}_{2}$, lipids and melanin, as well as exogenous contrast based on nonspecific dyes and specific targeting agents ${ }^{46,65}$, at high resolution. Spectral unmixing allows the contributions of these various contrast agents to be separated, enabling the simultaneous imaging of different markers of health and disease. In the present work, we demonstrate the simultaneous imaging of vasculature and gold nanoparticles at the level of entire tumours. This raises intriguing possibilities for tumour analysis that aims to correlate, for example, oxygenation and the activity of specific subsets of tumour and host cells, such as tumour-associated macrophages, which can be tracked in vivo using specifically designed nanoparticles ${ }^{66}$. Perfusion of gold nanoparticles into tumours could be accessed longitudinally in a non-invasive manner at a level of intra-tumoural detail not previously achieved 
with other tomographic set-ups ${ }^{18,19,67}$ and with a wholetumour imaging volume inaccessible to intravital microscopy. This finding highlights the potential of MSOM for preclinical screening and assessment of anticancer therapeutics in vivo or in organoids. The technique's combination of penetration depth, resolution, whole-tumour imaging, and non-invasiveness may give it advantages over intravital microscopy as a key tool in the drug discovery process ${ }^{68,69}$, such as for clarifying why nanoparticle-based cancer treatment often fails to improve therapeutic outcomes ${ }^{70}$.

Future efforts to improve the current MSOM system should focus on reducing measurement time using lasers with high repetition rates and fast wavelength scanning. Future work will introduce more image analysis methods to improve the quantitative performance of MSOM in tumour heterogeneity analysis.

Overall, MSOM is shown to image entire tumour morphology and physiology in vivo in a label-free, noninvasive manner at depths and resolutions never before demonstrated with an optical or optoacoustic method. By using a transducer array at a $15 \mathrm{MHz}$ central frequency and applying frequency equalization, our method allowed unprecedented cross-sectional imaging and achieved resolutions never before achieved in whole-tumour imaging by an optical contrast imaging method. As a result, we were able to quantify patterns of heterogeneity at the entire-tumour level and differentially quantify patterns of heterogeneity within parts of the tumour, such as the core and the rim. With its demonstrated abilities and the potential for hardware and software improvement, MSOM may prove to be a uniquely powerful preclinical technology on the road towards precision oncology, which aims to characterize tumour heterogeneity in order to select the most appropriate therapies and optimize prognosis.

\section{Materials and methods}

\section{In vivo tumour models}

Animal procedures were approved by the Government of Upper Bavaria. Female athymic Foxn1 nude mice (5week old, Envigo, Germany) were injected into the mammary fat pad with $4 \mathrm{~T} 1$ murine breast cancer cells (CRL-2539, ATCC, Manassas, VA, USA; $1 \times 10^{4}$ cells/ animal) or KPL4 human breast cancer cells ${ }^{71}$ (kindly provided by J. Kurebayashi, Kawasaki Medical School, Kurashiki, Japan; $2 \times 10^{6}$ cells/animal). NOD/SCIDSHrN nude mice (5-week old, Charles River Laboratories, Germany) were injected into the mammary fat pad with MDAMB-231 human breast cancer cells (CRM-HTB-26, ATCC; $3 \times 10^{6}$ cells/animal). Each type of cancer cell was injected into three animals under isoflurane anaesthesia. When tumours reached a diameter of $\sim 8 \mathrm{~mm}$, the animals were anaesthetized and imaged using MSOM. This tumour size was reached within 7-10 days in 4T1-injected animals, within approximately 40 days in KPL4-injected animals, and in 30-40 days in MDA-MB-231-injected animals. At this time, a mouse carrying a 4T1 tumour was injected intravenously with $50 \mu \mathrm{l}$ of gold nanoparticles (D12M-780-50, Nanopartz, USA) suspended in $200 \mu \mathrm{l}$ of phosphatebuffered saline (PBS) and then imaged in vivo.

\section{MSOM setup}

Figure 1a shows the custom-built MSOM system used for all experiments. The system features a conical scanning geometry for in vivo tumour imaging ${ }^{28,31,72,73}$. Single motorized stages for rotation (M-062. PD, Physik Instrumente, Germany) and translation (M-605.2DD, Physik Instrumente) allow scanning in discrete or continuous modes ${ }^{31}$. In the present study, in vivo imaging was performed in continuous scanning mode with an angle range of $255^{\circ}$ and a translation range of $10 \mathrm{~mm}$. Samples were excited optically using an optical parametric oscillator laser (Phocus II, Opotek, USA) tunable from 690 to $900 \mathrm{~nm}$ and capable of providing sub-10 ns pulses at a $10 \mathrm{~Hz}$ repetition rate and maximum pulse energy of $80 \mathrm{~mJ}$ at $760 \mathrm{~nm}$. The laser beam was delivered to the sample using a custom-made bundle of 672 fibres (Ceramoptec, Germany) divided into four arms, each of which measured $6.5 \times 2.5 \mathrm{~mm}$ and was positioned on a different side of the sample. This setup allowed homogeneous illumination of a volume of approximately $14 \times$ $14 \times 6 \mathrm{~mm}$. Laser pulse energies were selected based on sample size and were always between 30 and $50 \mathrm{~mJ}$ to remain below the ANSI-defined limit for biological samples. The average laser power was measured before each experiment at each wavelength using a Vega power metre (Ophir Photonics, Israel) to allow light fluence correction.

A sample holder was custom-designed to accommodate an entire mouse as well as the four heads of the fibre bundle, which were positioned at an angle of $8^{\circ}$ from the $x y$ plane. The holder was attached to a linear stage (NRT150, Thorlabs, Newton, NJ, USA) to allow adjustment of the holder position along the $z$ axis. The anaesthetized mouse was placed on top of the holder such that the tumour protruded through a hole and into a water bath held at $33^{\circ} \mathrm{C}$. The fibre bundle heads and transducer array were also immersed in the water bath. This bath provided acoustic coupling between the tumour and the transducer array. Snap rings with the same outer diameter and different inner diameters were fitted onto the hole according to tumour size in order to optimize sample placement and minimize the generation of acoustic signals from neighbouring tissues.

The ultrasound response from the sample was detected using a custom-made linear transducer array (Vermon, France) with 96 elements, a $15 \mathrm{MHz}$ central frequency and an average $-6 \mathrm{~dB}$ pulse-echo bandwidth of $45 \%$. Each 
element has a pitch of $100 \mu \mathrm{m}$, an elevation of $1.5 \mathrm{~mm}$ and a cylindrically focused feature with a focal length of $7.8 \mathrm{~mm}$. The array is mounted at $45^{\circ}$ on a custom-made tilting module connected to the translation-rotation scanner, allowing conical scanning around the tumour sample. The radius of the central plane traced by the central (48th) element in the array was $5.8 \mathrm{~mm}$. The linear array rotated and translated continuously at speeds chosen to provide optimal sample coverage at the $10 \mathrm{~Hz}$ repetition rate of the laser. Between two successive laser pulses, the array rotated by $\sim 0.035^{\circ}$ and translated $\sim 200 \mathrm{~nm}$.

\section{Calibration of the MSOM system}

The MSOM system was used to visualize a phantom consisting of black polyethylene microspheres with a diameter of $20 \mu \mathrm{m}$ (BKPMS 20-27, Cospheric, USA) uniformly dispersed in agar gel cylinders with a diameter of $12 \mathrm{~mm}$. These absorbers generate broadband optoacoustic signals with a frequency band wider than the maximum bandwidth of the linear array for the given laser pulse duration. The data collected with this phantom were used to calibrate the MSOM set-up, to measure the average speed of sound and to determine the spatial resolution of the system.

\section{Data acquisition and image reconstruction}

Approximately $7000 \times 96$ optoacoustic signals were acquired at each wavelength and digitized using a custombuilt data acquisition card (Falkenstein, Germany) operating at $10 \mathrm{~Hz}$ and sampling at $125 \mathrm{MS} / \mathrm{s}$ with 12-bit resolution over a $16 \mathrm{mV}$ range. The total scanning time per wavelength was $12 \mathrm{~min}$.

Ultrasound data collected after excitation at five wavelengths were used for tumour image reconstruction (Fig. 1c). Raw data were bandpass-filtered using a third-order Butterworth filter $(1-28 \mathrm{MHz})$ to remove noise and then passed through a time-variant filter ${ }^{74}$ to compensate for frequency-dependent acoustic attenuation. Threedimensional images were reconstructed using a backprojection algorithm ${ }^{75}$ with a voxel size of $24 \times 24 \times$ $24 \mu \mathrm{m}$. Reconstructed images were corrected for light fluence as previously described ${ }^{76}$. Frequency equalization was performed by reconstructing optoacoustic images in two frequency bands of $1-7.5 \mathrm{MHz}$ and $4-28 \mathrm{MHz}$, and the image features from each band were coloured differently and overlaid using Image $\mathrm{J}^{77}$. Finally, linear spectral unmixing $^{29,78}$ was used to generate separate reconstructions of the distribution of endogenous or exogenous contrast agents. The unmixing algorithm assumed a constant background spectrum and absorption spectra of $\mathrm{Hb}, \mathrm{HbO}_{2}$, and gold nanoparticles (Fig. 1c). Unmixed images were generated and analysed using ImageJ. For display purposes, the gamma function in ImageJ was used in some figures to highlight information from the tumour core, but all quantitative analyses were performed on unmixed results without further processing. The unmixing algorithm provides values $\left(\mathrm{Hb}, \mathrm{HbO}_{2}\right.$ and gold nanoparticles) that are in arbitrary units and relative across different structures in the reconstructed images. The $\mathrm{sO}_{2}$ values are relative rather than absolute, as they are calculated as ratios from the $\mathrm{Hb}$ and $\mathrm{HbO}_{2}$ values.

\section{Tumour heterogeneity analysis and tumour segmentation}

The presented metrics were calculated by averaging the metric values in MIPs of $400 \mu \mathrm{m}$ sections at different tumour depths throughout the entire tumour. Variance, skewness, kurtosis, energy and entropy were calculated as previously described ${ }^{37}$. Coarseness, contrast and directionality were calculated using Tamura texture analysis ${ }^{36}$. The centre and rim regions of tumours were partitioned using cut-off points identified from the lines connecting the centre point and boundary points. Relative differences were calculated as the ratio between a metric's absolute difference between the centre and rim to the metric's sum for the centre and rim.

\section{Spectral error maps}

We assessed the reliability of MSOM results by calculating fitting residuals describing the pixel-wise deviations between the reconstructed multi-spectral image and the corresponding linear unmixed signal. The MIP across 20 slices of the signal norm was computed, and each pixel of the resulting multispectral image was fitted to the spectra of $\mathrm{Hb}$ and $\mathrm{HbO}_{2}$ or gold nanoparticles. Then, the norm of the residuals relative to the norm of the signal was displayed as an image to identify the relative uncertainty associated with different parts of the tumour image (SI Appendix, Fig. S3).

\section{Tumour histology, immunohistochemistry, and lectin staining}

Cryosections $(10 \mu \mathrm{m}$ thick) were prepared using standard procedures (CM1950Cryostat, Leica). For haematoxylin-eosin staining, sections were thawed, dried on a heating plate, fixed in paraformaldehyde and stained with haematoxylin (30 s) and eosin (1 s) using standard procedures. For immunohistochemistry, sections were dried on a heating plate, fixed in cold acetone for $10 \mathrm{~min}$, and then rinsed in Tris-buffered saline containing 0.3\% Triton X-100 (TBST). Slices were blocked for $1 \mathrm{~h}$ at room temperature with TBST containing $10 \%$ goat serum. The blocking solution was removed, and sections were immunostained overnight at $4{ }^{\circ} \mathrm{C}$ with a rat primary antibody against mouse CD31 (1:20; DIA-310, Dianova, Hamburg, Germany) to show the blood vessel distribution. Sections were washed three times (10 min each) with TBST, incubated for $1 \mathrm{~h}$ at room temperature with an Alexa Fluor 594-conjugated goat anti-rat secondary 
antibody (1:200; A-11007, Thermo Fisher Scientific, Munich, Germany), and then washed again three times (10 min each) with TBST. Alternatively, sections were immunostained against HIF- $1 \alpha$ as described above except using a rabbit primary antibody against mouse HIF- $1 \alpha$ (1:50; ab2185, Abcam, Cambridge, UK) and an Alexa Fluor 488-conjugated goat anti-rabbit secondary antibody (1:200; A-11034, Thermo Fisher Scientific). All antibodies were diluted in TBST containing $1 \%$ goat serum. Sections were mounted using ProLong Gold Antifade Reagent with DAPI (catalogue no. 8961S, Cell Signalling Technology, MA, USA) and analysed using a Zeiss Axio Imager M2 microscope and Zeiss ZEN 2 Pro (Blue edition) software.

In some experiments, animals were injected intravenously with pimonidazole- $\mathrm{HCl}(80 \mathrm{mg} / \mathrm{kg})$ from the Hypoxyprobe Kit (Hypoxyprobe, Burlington, MA, USA) and sacrificed $1 \mathrm{~h}$ later. Pimonidazole distributes to all tissues and forms adducts with thiol-containing proteins in cells where the oxygen partial pressure is below approximately $10 \mathrm{mmHg}$ at $37^{\circ} \mathrm{C}^{79-81}$. Tumour sections were prepared and immunostained as described above using the mouse primary monoclonal antibody supplied in the Hypoxyprobe Kit and an Alexa Fluor 594conjugated goat anti-mouse secondary antibody (1:200; A-11005, Thermo Fisher Scientific).

\section{Profile, histogram and Hamming distance}

Profiles in Fig. 3g, h were obtained along the white lines indicated in Fig. 3a, d and Fig. 3b, e. These line profiles were smoothed by the moving mean function (in MATLAB) with window lengths of 60 and 240. The histogram with a total of 256 bins was constructed in the undersampled images of $256 \times 256$ pixels with background subtraction. Undersampling was performed using spline interpolation in Fig. 3a-f.

Based on an image's visual appearance, a discrete cosine transform (DCT)-based image hashing algorithm can produce a hash value for each image stored in an unsigned 64-bit integer ${ }^{35}$. Hamming distance can be used to measure the differences between two hash values within the range from 0 to 64 and then identify whether two images are perceptually different or similar. The maximum Hamming distance of 32 in Fig. $3 k-m$ indicates that at most half of the elements in the two hash values are different. If a pre-defined threshold is set to 32.00 and if both images have Hamming distances smaller than this threshold in pHash, then the two images can be considered perceptually similar.

\section{Acknowledgements}

We thank Dr. Yihan Wang and Dr. Korbinian Paul-Yuan for their help with fluence correction and spectral error maps. We also thank A. Chapin Rodríguez, $\mathrm{PhD}$, for helpful discussions. The research leading to these results has received funding from the Deutsche Forschungsgemeinschaft (DFG), Germany [Gottfried Wilhelm Leibniz Prize 2013; NT 3/10-1]. This project has received funding from the European Research Council (ERC) under the European Union's Horizon 2020 research and innovation programme under grant agreement 694968 (PREMSOT). This work was also funded by the National Natural Science Foundation of China (81771880, 81401453). J.L. acknowledges the support of a China Scholarship Council grant (201506255001). J.P. acknowledges support from the Alexander von Humboldt Postdoctoral Fellowship Program.

\section{Author details}

${ }^{1}$ School of Precision Instruments and Optoelectronics Engineering, Tianjin University, No.92, Weijin Road, Nankai District, 300072 Tianjin, China. ${ }^{2}$ Institute of Biological and Medical Imaging, Helmholtz Zentrum München, Ingolstädter Landstr. 1, D-85764 Neuherberg, Germany. ${ }^{3}$ Chair of Biological Imaging, TranslaTUM, Technische Universität München, Ismaningerstr. 22, D-81675 Munich, Germany. ${ }^{4}$ Department of Instrumentation and Applied Physics, Indian Institute of Science Bangalore, CV Raman Rd, Bengaluru 560012 Karnataka, India

\section{Author contributions}

J.L. and V.N. conceived the project. J.L. and A.C. worked on the MSOM system. J.L., S.G., V.G. and M.O. designed imaging experiments, J.L., J.P., P.V., B.K., and I.O. implemented image reconstruction and data analyses. J.L. and J.P. wrote the manuscript. V.N. supervised the research and edited the manuscript. All authors revised the manuscript and commented on the content.

Conflict of interest

The authors declare that they have no conflict of interest.

Supplementary information is available for this paper at https://doi.org/ 10.1038/s41377-020-0295-y.

Received: 28 August 2019 Revised: 10 February 2020 Accepted: 19 March 2020

Published online: 13 April 2020

\section{References}

1. Martin, J. D. et al. Reengineering the tumor microenvironment to alleviate hypoxia and overcome cancer heterogeneity. Cold Spring Harb. Perspect. Med. 6, a027094 (2016)

2. Alizadeh, A. A. et al. Toward understanding and exploiting tumor heterogeneity. Nat. Med. 21, 846-853 (2015).

3. Vennin, $C$. et al. Intravital imaging reveals new ancillary mechanisms co-opted by cancer cells to drive tumor progression. F1000Research 5, 892 (2016).

4. Ellenbroek, S. I. J. \& Van Rheenen, J. Imaging hallmarks of cancer in living mice. Nat. Rev. Cancer 14, 406-418 (2014).

5. Nobis, M. et al. Advanced intravital subcellular imaging reveals vital threedimensional signalling events driving cancer cell behaviour and drug responses in live tissue. FEBS J. 280, 5177-5197 (2013).

6. Maeda, A., Kulbatski, I. \& DaCosta, R. S. Emerging applications for optically enabled intravital microscopic imaging in radiobiology. Mol. Imaging 14, 452-474 (2015).

7. Ntziachristos, V. Going deeper than microscopy: the optical imaging frontier in biology. Nat. Methods 7, 603-614 (2010).

8. Yao, J. J. et al. Label-free oxygen-metabolic photoacoustic microscopy in vivo. J. Biomed. Opt. 16, 076003 (2011)

9. Garvalov, B. K. \& Ertürk, A. Seeing whole-tumour heterogeneity. Nat. Biomed. Eng. 1, 772-774 (2017).

10. Alieva, M. et al. Imaging windows for long-term intravital imaging: general overview and technical insights. IntraVital 3, e29917 (2014).

11. Dobosz, M. et al. Multispectral fluorescence ultramicroscopy: threedimensional visualization and automatic quantification of tumor morphology, drug penetration, and antiangiogenic treatment response. Neoplasia 16 1-13 (2014)

12. Tanaka, N. et al. Whole-tissue biopsy phenotyping of three-dimensional tumours reveals patterns of cancer heterogeneity. Nat. Biomed. Eng. 1, 796-806 (2017).

13. Hai, P. F. et al. High-throughput, label-free, single-cell photoacoustic microscopy of intratumoral metabolic heterogeneity. Nat. Biomed. Eng. 3, 381-391 (2019). 
14. Mallidi, S., Luke, G. P. \& Emelianov, S. Photoacoustic imaging in cancer detection, diagnosis, and treatment guidance. Trends Biotechnol. 29, 213-221 (2011).

15. Omar, M., Aguirre, J. \& Ntziachristos, V. Optoacoustic mesoscopy for biomedicine. Nat. Biomed. Eng. 3, 354-370 (2019).

16. Taruttis, A., Van Dam, G. M. \& Ntziachristos, V. Mesoscopic and macroscopic optoacoustic imaging of cancer. Cancer Res. 75, 1548-1559 (2015).

17. Ermolayev, V. et al. Simultaneous visualization of tumour oxygenation, neovascularization and contrast agent perfusion by real-time three-dimensional optoacoustic tomography. Eur. Radiol. 26, 1843-1851 (2016).

18. Herzog, E. et al. Optical imaging of cancer heterogeneity with multispectral optoacoustic tomography. Radiology 263, 461-468 (2012).

19. Tomaszewski, M. R. et al. Oxygen enhanced optoacoustic tomography (OE-OT) reveals vascular dynamics in murine models of prostate cancer. Theranostics $\mathbf{7}$, 2900-2913 (2017)

20. Jathoul, A. P. et al. Deep in vivo photoacoustic imaging of mammalian tissues using a tyrosinase-based genetic reporter. Nat. Photonics 9 239-246 (2015).

21. Laufer, J. G. et al. In vivo preclinical photoacoustic imaging of tumor vasculature development and therapy. J. Biomed. Opt. 17, 056016 (2012).

22. Omar, M. et al. Pushing the optical imaging limits of cancer with multifrequency-band raster-scan optoacoustic mesoscopy (RSOM). Neoplasia 17, 208-214 (2015).

23. Ruan, Q. et al. Development of an anti-angiogenic therapeutic model combining SCAAV2-delivered siRNAs and noninvasive photoacoustic imaging of tumor vasculature development. Cancer Lett. 332, 120-129 (2013).

24. Zhang, H. F. et al. Functional photoacoustic microscopy for high-resolution and noninvasive in vivo imaging. Nat. Biotechnol. 24, 848-851 (2006).

25. Diot, G. et al. Multispectral optoacoustic tomography (MSOT) of human breast cancer. Clin. Cancer Res. 23, 6912-6922 (2017).

26. Lao, Y. Q. et al. Noninvasive photoacoustic imaging of the developing vasculature during early tumor growth. Phys. Med. Biol. 53, 4203-4212 (2008).

27. Siphanto, R. I. et al. Serial noninvasive photoacoustic imaging of neovascularization in tumor angiogenesis. Opt. Express 13, 89-95 (2005).

28. Chekkoury, A. et al. High-resolution multispectral optoacoustic tomography of the vascularization and constitutive hypoxemia of cancerous tumors. Neoplasia 18, 459-467 (2016).

29. Razansky, D. et al. Multispectral opto-acoustic tomography of deep-seated fluorescent proteins in vivo. Nat. Photonics 3, 412-417 (2009).

30. Ntziachristos, V. \& Razansky, D. Molecular imaging by means of multispectral optoacoustic tomography (MSOT). Chem. Rev. 110, 2783-2794 (2010).

31. Gateau, J., Chekkoury, A. \& Ntziachristos, V. High-resolution optoacoustic mesoscopy with a $24 \mathrm{MHz}$ multidetector translate-rotate scanner. J. Biomed. Opt. 18, 106005 (2013)

32. Aguirre, J. et al. Precision assessment of label-free psoriasis biomarkers with ultra-broadband optoacoustic mesoscopy. Nat. Biomed. Eng. 1, 0068 (2017).

33. Miettinen, M. Immunohistochemistry of soft tissue tumours - review with emphasis on 10 markers. Histopathology 64, 101-118 (2014).

34. Bos, R. et al. Levels of hypoxia-inducible factor-1 a during breast carcinogenesis. J. Natl Cancer Inst. 93, 309-314 (2001).

35. Zauner, C. Implementation and benchmarking of perceptual image hash functions. Master's thesis, Upper Austria University of Applied Sciences, Hagenberg Campus (2010).

36. Tamura, H., Mori, S. \& Yamawaki, T. Textural features corresponding to visual perception. IEEE Trans. Syst., Man, Cybern. 8, 460-473 (1978)

37. Gonzalez, R. C. \& Woods, R. E. Digital Image Processing. 3rd edn. 828-836 (Upper Saddle River: Prentice Hall, 2008)

38. Yoon, S. H. et al. Tumor heterogeneity in lung cancer: assessment with dynamic contrast-enhanced MR imaging. Radiology 280, 940-948 (2016).

39. Chicklore, S. et al. Quantifying tumour heterogeneity in ${ }^{18} \mathrm{~F}$-FDG PET/CT imaging by texture analysis. Eur. J. Nucl. Med. Mol. Imaging 40, 133-140 (2013).

40. Brooks, F. J. On some misconceptions about tumor heterogeneity quantification. Eur. J. Nucl. Med. Mol. Imaging 40, 1292-1294 (2013).

41. Baek, H. J. et al. Percent change of perfusion skewness and kurtosis: a potential imaging biomarker for early treatment response in patients with newly diagnosed glioblastomas. Radiology 264, 834-843 (2012).

42. Yun, B. L. et al. Intratumoral heterogeneity of breast cancer xenograft models: texture analysis of diffusion-weighted MR imaging. Korean J. Radiol. 15, 591-604 (2014).
43. Miles, K. A., Ganeshan, B. \& Hayball, M. P. CT texture analysis using the filtrationhistogram method: what do the measurements mean? Cancer Imaging 13, 400-406 (2013).

44. Weiss, G. J. et al. Noninvasive image texture analysis differentiates K-ras mutation from pan-wildtype NSCLC and is prognostic. PLOS ONE 9, e100244 (2014).

45. Di Pietro, P. et al. Gold and silver nanoparticles for applications in theranostics. Curr. Top. Medicinal Chem. 16, 3069-3102 (2016).

46. Gujrati, V., Mishra, A. \& Ntziachristos, V. Molecular imaging probes for multispectral optoacoustic tomography. Chem. Commun. 53, 4653-4672 (2017).

47. Albini, A. et al. Cancer prevention by targeting angiogenesis. Nat. Rev. Clin Oncol. 9, 498-509 (2012).

48. Pulaski, B. A. \& Ostrand-Rosenberg, S. Reduction of established spontaneous mammary carcinoma metastases following immunotherapy with major histocompatibility complex class II and B7.1 cell-based tumor vaccines. Cancer Res. 58, 1486-1493 (1998).

49. Molina, D. et al. Lack of robustness of textural measures obtained from 3D brain tumor MRIs impose a need for standardization. PLOS ONE 12, e0178843 (2017).

50. Wagner, F. et al. Comparison of contrast-enhanced $C T$ and $\left[{ }^{18} \mathrm{~F}\right] \mathrm{FDG}$ PET/CT analysis using kurtosis and skewness in patients with primary colorectal cancer. Mol. Imaging Biol. 19, 795-803 (2017).

51. Chaudhury, B. et al. Heterogeneity in intratumoral regions with rapid gadolinium washout correlates with estrogen receptor status and nodal metastasis. J. Magn. Reson. Imaging 42, 1421-1430 (2015).

52. Cook, G. J. R. et al. Characterisation of malignant peripheral nerve sheath tumours in neurofibromatosis-1 using heterogeneity analysis of ${ }^{18}$ F-FDG PET. Eur. J. Nucl. Med. Mol. Imaging 44, 1845-1852 (2017).

53. Doshi, A. M. et al. Use of MRI in differentiation of papillary renal cell carcinoma subtypes: qualitative and quantitative analysis. Am. J. Roentgenol. 206, 566-572 (2016).

54. Li, H. et al. Quantitative MRI radiomics in the prediction of molecular classifications of breast cancer subtypes in the TCGAVTCIA data set. npj Breast Cancer 2, 16012 (2016).

55. Shen, W. C. et al. [18]Fluorodeoxyglucose positron emission tomography for the textural features of cervical cancer associated with lymph node metastasis and histological type. Eur. J. Nucl. Med. Mol. Imaging 44, 1721-1731 (2017).

56. Soussan, M. et al. Relationship between tumor heterogeneity measured on FDG-PET/CT and pathological prognostic factors in invasive breast cancer. PLOS ONE 9, e94017 (2014).

57. Rodriguez Gutierrez, D. et al. Serial MR diffusion to predict treatment response in high-grade pediatric brain tumors: a comparison of regional and voxelbased diffusion change metrics. Neuro-Oncol. 15, 981-989 (2013).

58. Tochigi, T. et al. Heterogeneity of glucose metabolism in esophageal cancer measured by fractal analysis of fluorodeoxyglucose positron emission tomography image: correlation between metabolic heterogeneity and survival. Digestive Surg. 34, 186-191 (2017).

59. Zhou, M. et al. Identifying spatial imaging biomarkers of glioblastoma multiforme for survival group prediction. J. Magn. Reson. Imaging 46, 115-123 (2017).

60. Hayano, K. et al. Fractal analysis of contrast-enhanced $\subset T$ images to predict survival of patients with hepatocellular carcinoma treated with sunitinib. Digestive Dis. Sci. 59, 1996-2003 (2014).

61. Aerts, H. J. W. L. et al. Decoding tumour phenotype by noninvasive imaging using a quantitative radiomics approach. Nat. Commun. 5, 4006 (2014).

62. Chitnis, P. V., Mamou, J. \& Feleppa, E. J. Spectrum analysis of photoacoustic signals for characterizing lymph nodes. J. Acoustical Soc. Am. 135, 2372 (2014)

63. Patterson, M. P. et al. Optoacoustic characterization of prostate cancer in an in vivo transgenic murine model. J. Biomed. Opt. 19, 056008 (2014).

64. $\mathrm{Xu}, \mathrm{G}$. et al. The functional pitch of an organ: quantification of tissue texture with photoacoustic spectrum analysis. Radiology 271, 248-254 (2014).

65. Weber, J., Beard, P. C. \& Bohndiek, S. E. Contrast agents for molecular photoacoustic imaging. Nat. Methods 13, 639-650 (2016).

66. Cuccarese, M. F. et al. Heterogeneity of macrophage infiltration and therapeutic response in lung carcinoma revealed by $3 \mathrm{D}$ organ imaging. Nat. Commun. 8, 14293 (2017).

67. Li, M. L. et al. Simultaneous molecular and hypoxia imaging of brain tumors in vivo using spectroscopic photoacoustic tomography. PrOc. IEEE 96, 481-489 (2008). 
68. Conway, J. R. W., Carragher, N. O. \& Timpson, P. Developments in preclinical cancer imaging: innovating the discovery of therapeutics. Nat. Rev. Cancer 14 314-328 (2014).

69. Weissleder, R. et al. Imaging approaches to optimize molecular therapies. Sci. Transl. Med. 8, 355ps16 (2016).

70. Miller, M. A. \& Weissleder, R. Imaging the pharmacology of nanomaterials by intravital microscopy: toward understanding their biological behavior. Adv. Drug Deliv. Rev. 113, 61-68 (2017).

71. Kurebayashi, J. et al. Isolation and characterization of a new human breast cancer cell line, KPL-4, expressing the Erb B family receptors and interleukin-6. Br. J. Cancer 79, 707-717 (1999).

72. Chekkoury, A. et al. Optical mesoscopy without the scatter: broadband multispectral optoacoustic mesoscopy. Biomed. Opt. Express 6, 3134-3148 (2015).

73. Gateau, J., Chekkoury, A. \& Ntziachristos, V. Ultra-wideband three-dimensional optoacoustic tomography. Opt. Lett. 38, 4671-4674 (2013).

74. Treeby, B. E. Acoustic attenuation compensation in photoacoustic tomography using time-variant filtering. J. Biomed. Opt. 18, 036008 (2013).
75. Gateau, J. et al. Three-dimensional optoacoustic tomography using a conventional ultrasound linear detector array: whole-body tomographic system for small animals. Med. Phys. 40, 013302 (2013).

76. Jetzfellner, T. et al. Performance of iterative optoacoustic tomography with experimental data. Appl. Phys. Lett. 95, 013703 (2009).

77. Schneider, C., Rasband, W. \& Eliceiri, K. NIH Image to ImageJ: 25 years of image analysis. Nat. Methods 9, 671-675 (2012).

78. Tzoumas, S. et al. Unmixing molecular agents from absorbing tissue in multispectral optoacoustic tomography. IEEE Trans. Med. Imaging 33, 48-60 (2014).

79. Varia, M. A. et al. Pimonidazole: a novel hypoxia marker for complementary study of tumor hypoxia and cell proliferation in cervical carcinoma. Gynecologic Oncol. 71, 270-277 (1998).

80. Arteel, G. E. et al. Evidence that hypoxia markers detect oxygen gradients in liver: pimonidazole and retrograde perfusion of rat liver. Br. J. Cancer $\mathbf{7 2}$, 889-895 (1995)

81. Kennedy, A. S. et al. Proliferation and hypoxia in human squamous cell carcinoma of the cervix: first report of combined immunohistochemical assays. Int. J. Radiat. Oncol. Biol. Phys. 37, 897-905 (1997). 